\title{
Identification of Resistance Sources to Wheat Stem Rust from Introduced Genotypes in Kenya
}

\author{
Molly O. Akello ${ }^{1}$, Felister Nzuve ${ }^{1}$, Florence Olubayo ${ }^{1}$, Godwin Macharia ${ }^{2} \&$ James Muthomi $^{1}$ \\ ${ }^{1}$ Department of Plant Science and Crop Protection, University of Nairobi, Nairobi, Kenya \\ ${ }^{2}$ Kenya Agricultural and Livestock Research Organization, Njoro, Kenya \\ Correspondence: Molly O. Akello, Department of Plant Science and Crop Protection, University of Nairobi, P.O. \\ Box 29053-0625, Nairobi, Kenya. Tel: 254-(0)-712-140-781. E-mail: mollyakello2@gmail.com
}

Received: October 18, $2016 \quad$ Accepted: November 25, $2016 \quad$ Online Published: January 15, 2017

doi:10.5539/jas.v9n2p73 URL: http://dx.doi.org/10.5539/jas.v9n2p73

The research is financed by Alliance for a Green Revolution in Africa (AGRA).

\begin{abstract}
Stem rust Puccinia graminis Pers. f. sp. tritici of wheat is the most important disease in Kenya. Emergence of race Ug99 and other variants virulent to host resistance genes including $\mathrm{Sr} 31$ has rendered $95 \%$ of Kenyan cultivars susceptible. This study aimed to identify new sources of resistance to stem rust in a collection of exotic genotypes. Three hundred and sixteen wheat genotypes were screened at the Kenya Agricultural and Livestock Research Organization (KALRO) in Njoro for two seasons in 2015. The host reaction to disease was evaluated based on the modified Cobb scale. The relative Final Rust Severity (rFRS), Average Coefficient of Infection (ACI) and relative Area Under Disease Progress Curve (rAUDPC) were used to characterize the genotypes for stem rust resistance. Agronomic traits were also recorded. Six genotypes namely ALBW-100, ALBW- 204, EPCBW-261, EPCBW-295, PCHP-309 and PCHPBW-310 with significantly low ACI, rAUDPC and rFRS were identified. Thirty five genotypes showed Pseudo-Black Chaff (PBC) phenotype associated with resistant gene $S r 2$, a source of partial resistance in wheat. The genotypes also showed low disease severity $(20-25 \%)$ and Moderately Susceptible (MS) - Susceptible (S) infection types in both seasons. Genotypes had significant differences $(p \leq 0.05)$ on plant height, 1000-kernel weight and number of tillers indicating genetic variation which could be exploited in breeding for resistance to stem rust. The negative relationship between agronomic variables involving plant height, spikelet length and 1000-kernel weight showed harmful effects of stem rust on plant characteristics including yield. The stem rust resistant genotypes with good agronomic traits could be introgressed into adapted Kenyan backgrounds while the genotypes showing presence of PBC could be utilized to develop durable stem rust resistant wheat. Inheritance studies to elucidate the exact genes conferring resistance to stem rust could be conducted for breeders to exploit their genetic variability.
\end{abstract}

Keywords: adult plant resistance (APR), introduced genotypes, $S r 2$ genes, stem rust

\section{Introduction}

Wheat (Triticum aestivum spp. aestivum L.) is an important food grain source worldwide. Its demand in developing countries is rising in recent decades and is expected to reach $60 \%$ by 2050 (FAO, 2016). However, climate change associated with erratic temperatures, droughts, floods, pests and disease epidemics will reduce wheat production by $29 \%$ (Rosegrant et al., 1995). Ever since wheat was introduced in Kenya-nearly a century ago, stem rust has remained a key production challenge. Strategic introgression and deployment of resistance genes in commercial varieties throughout the 1950s greatly circumvented major stem rust epidemics. However, in more recent years, the evolution and selection for new races with increased virulence has become undesirably frequent (Velu \& Singh, 2013). In the last decade, the threats due to stem rust disease have re-emerged owing to a new set of races, called the "Ug99 family". The first recognizable variant in this family is the initially characterized race Ug99, also designated as TTKSK, based on its effects on select host resistance genes differentials (Jin et al., 2008). First reported in Uganda in 1998, the race TTKSK was found in Kenya in 2001. This race has unique virulence to $\operatorname{Sr} 31$ and $\operatorname{Sr} 38$ resistance genes widely utilized in wheat worldwide and for which virulence had not been reported previously in the world (Pretorius et al., 2000). 
Globally, since the discovery of Ug99, about $80 \%$ to $90 \%$ of the wheat grown is susceptible to stem rust often leading to up to $70 \%$ yield losses (Singh et al., 2006). In Kenya, stem rust associated yield losses of $100 \%$, have been reported (Njau et al., 2010). Over $95 \%$ of local commercial varieties are susceptible or highly susceptible, while only a few older varieties showing some level of 'adult plant resistance' (APR) (Wanyera et al., 2006; Njau et al., 2010) have been identified. Currently, more than 15 confirmed races in the Ug99 lineage have been reported in Africa and beyond (Singh et al., 2015). Through airborne transmission, Ug99 and its variants have reached Asia one of the main global wheat bread baskets; having been reported in Yemen in 2006, Iran in 2007 and Pakistan in 2009 (Hodson et al., 2009; Nazari et al., 2009; Admassu et al., 2009).

While chemicals can be used to manage stem rust, the main challenge is the high costs and the detrimental effects posed on the environment (Beard et al., 2006). Genetic resistance is the most economically viable method of controlling stem rust. To date over 70 genes have been designated for resistance to stem rust (McIntosh et al., 2014). Of those, 34 are ineffective against race Ug99 (Singh et al., 2015). Among the adult plant resistance genes, $\mathrm{Sr} 2$ gene is the only well studied. A combination of $\mathrm{Sr} 2$ gene and other unknown slow rusting resistance genes forms the "Sr2 complex" which provides durable resistance to stem rust (McIntosh, 1988). The concept-"breeding for durable resistance in wheat" championed by Dr. Norman Borlaug has led to a global search for new genes or gene combinations to combat the new stem rust race Ug99 that could be released as new varieties.

A shuttle breeding scheme with the goal of phenotyping breeding populations for adult plant resistance to Ug99 family of races was initiated in 2005 between KALRO-Njoro and the International Maize and Wheat Improvement Centre (CIMMYT) (http://www.globalrust.org/). Through the initiative, several genotypes, with potentially good sources of APR genes have been identified. A study conducted at KALRO-Njoro on elite advanced CIMMYT bread wheat indicated that $30 \%$ of the materials were susceptible at the seedling stage while various levels of APR were identified in the field tests (Njau et al., 2010). Invariably, all APR in the studied material was associated with the $S r 2$ complex. Bhavani et al. (2011) reported detection of $S r 2$ gene based on mapping studies on six CIMMYT parental lines. These reports indicate that exotic wheat genotypes could be good sources of resistance to stem rust that could be deployed in the national wheat improvement program at KALRO-Njoro. A study on 30 vintage Kenyan varieties for resistance to stem rust both at the seedling and adult plant growth stage revealed that none of them except variety Bonny was resistant at the seedling stage, while a few had APR attributed to $S r 2$ gene in their backgrounds (Njau et al., 2009).

Given the devastating nature of $\mathrm{Ug} 99$ family of stem rust races to wheat productivity regionally, effort to explore for resistance sources and incorporation of effective genes into new high yield commercial varieties is paramount. Accordingly, the objective of this study was to identify suitable sources of resistance to stem rust among exotic wheat genotypes.

\section{Materials and Methods}

\subsection{Experimental Site}

The research was carried at KALRO-Njoro which is about 2185 meters above sea level, approximately $0^{\circ} 20^{\prime} \mathrm{S}$; $35^{\circ} 56^{\prime} \mathrm{E}$. Average temperatures ranges between $9.7^{\circ} \mathrm{C}$ and $23.5^{\circ} \mathrm{C}$. Mean annual precipitation is $900 \mathrm{~mm}$ (Ooro et al., 2009). KALRO-Njoro hosts the global phenotyping facility for characterizing and selection of wheat genotypes resistant to stem rust (Singh et al., 2006), under the auspices of the BGRI project (http://www.globalrust.org/).

\subsection{Genotypes}

Three hundred and sixteen exotic genotypes namely: 250 "Aluminum Bread Wheat (AL BW)", 47 "Elite Bread Wheat (EPC BW)" and 19 "PC-Harvest plus Bread Wheat (PCHP BW)". These are genetically fixed lines, bred targeting high yields and superior grain quality including specific nutritional needs (Velu \& Singh, 2013). The universally stem rust susceptible genotype CACUKE was included to monitor proliferation of the disease epidemic. A mixture of seven rust susceptible genotypes including Morocco, Robin, and PBW343 were used.

\subsection{Experimental Procedures}

The study was undertaken twice; off-season (January to June 2015) and main-season nursery (July to November 2015). Experiments in both seasons were established based on augmented alpha lattice square design with no replication. Experimental plots were 6 rows by $2 \mathrm{~m}$ length and $20 \mathrm{~cm}$ inter-row spacing. Diammonium phosphate (DAP) was applied at the rate of $125 \mathrm{~kg} / \mathrm{ha}$ while planting, followed by an application of Urea $(75 \mathrm{~kg} / \mathrm{ha})$ as a source of Nitrogenn at late tillering and booting stages of the plants. 


\subsection{Data Collection}

\subsubsection{Agronomic Traits}

These included: plant height $(\mathrm{cm})$, spike length $(\mathrm{cm})$ and 1000-kernel weight $(\mathrm{g})$ evaluated at harvest maturity. Presence of $S r 2$ gene was recorded by observing occurrence of pseudo-black chaff (PBC) on the stems and heads of the plant. A plus (+) was used to indicated presence of PBC while a minus (-) indicated absence of PBC.

\subsubsection{Disease Scoring}

This was done in season one and two when the susceptible check CACUKE expressed 50\% rust severity, and $70 \%$ severity respectively. The stem rust severity was recorded based on the modified Cobb's scale on a $0-100 \%$ scale (Peterson et al., 1948). The host response which included the trace (TR), resistant (R), moderately resistant (MR), moderately susceptible (MS), and susceptible (S) were recorded based on Roelfs et al. (1992) scale. The disease severity scores were converted into area under the disease progress curve (AUDPC) values following Wilcoxson et al. (1975) Equation 1.

$$
A U D P C=\sum_{i=1}^{n} \frac{\left(y_{i}+y_{i+1}\right)}{2}\left(t_{i+1}-t_{i}\right)
$$

Where, $y_{i}=$ average coefficient of infection of the $i^{\text {th }}$ reading; $y_{i+1}=$ average coefficient of infection of $i+1^{\text {th }}$ reading; $\left(t_{i+1}-t_{i}\right)=$ number of days between the $i^{\text {th }}$ and the $i+i^{\text {th }}$ reading, and $\mathrm{n}=$ number of observations.

The susceptible check CACUKE was used as a reference to obtain the relative AUDPC (rAUDPC) in Equation 2, as well as relative Final Rust Severity (rFRS), in Equation 3.

$$
\begin{aligned}
r A U D P C & =\frac{\text { AUDPC of Genotypes }}{\text { AUDPC of Susceptible Check }} \times 100 \\
r \text { Final Rust Severity } & =\frac{\text { Final Rust Severity of Genotype }}{\text { Final Rust Severity of SusceptibleCheck }} \times 100
\end{aligned}
$$

The coefficient of infection (CI) was obtained by multiplying the final disease severity for each individual score by the numerical value where $\mathrm{TR}=0.1 ; \mathrm{R}=0.2 ; \mathrm{MR}=0.4 ; \mathrm{M}=0.6 ; \mathrm{MS}=0.8$; and averaged to give average coefficient of infection (ACI) (Roelfs et al., 1992).

\subsection{Data Analysis}

The analysis of variance (ANOVA) was used to discern differences among genotypes. Genotypes were considered fixed while the seasons were considered as random effects. Genotypic means were separated based on Fisher's protected least significance difference (LSD) at $p \leq 0.05$. A Pearson correlation coefficient test was done to determine the relationship between the different disease parameters and the agronomic traits.

\section{Results}

\subsection{Reactions of Genotypes to Weather Conditions across the Seasons}

There was high disease pressure among the genotypes during both seasons. The susceptible check, CACUKE showed $90 \%$ and $100 \%$ final rust severity in season one and two respectively.

\subsection{Reaction of Genotypes to Stem Rust and Other Agronomic Traits}

ANOVA revealed significant differences at $p \leq 0.05$ among the genotypes for all the agronomic traits, apart from TKW (Table 1). Seasonal variations were significant at $(p \leq 0.05)$ for AUDPC, rAUDPC, TKW and PH. Significant genotypes $\times$ season interactions were revealed among the disease parameters but not the agronomic traits. Dwarf genotypes with heights ranging from 62.5 to $68.2 \mathrm{~cm}$ relative to the tallest genotype (ALBW-135) $94 \mathrm{~cm}$ tall were identified in both seasons. These were: ALBW-38, ALBW-65, ALBW-72, ALBW-81, ALBW-82, ALBW-99, ALBW-108, ALBW-207, ALBW-208, ALBW-210, EPCBW-292, PCHP-300, PCHP-312 and PCHP-316. They also recorded high TKW ranging between 32.7 to $39.2 \mathrm{~g}$ in both seasons (Table 2). The longest spikes averaged from $12.4 \mathrm{~cm}$ among the ALBW genotypes. ALBW-98 had the highest average TKW (47.4 g). Notably, rust susceptible genotypes ALBW-16 depicting high disease severities of $40 \%$ and $50 \%$ in season one and two respectively also had a relatively low average TKW of $24.4 \mathrm{~g}$, only slightly higher than that of the susceptible check CACUKE $20.2 \mathrm{~g}$ in season 2 (Table 2).

Genetic variations for resistance to stem rust within each season were observed. Six genotypes: ALBW-100, ALBW-204, EPCBW-261, EPCBW-295, PCHP-309 and PCHPBW-310, showed R-MR response in season one and trace responses to stem rust in season two. Among the ALBW, ACI ranged from 1-60, while the AUDPC ranged from 15-455 in season one and 0-795 in season two. Over half (54\%) of the two hundred and fifty ALBW recorded moderately susceptible-susceptible (MSS) response with AUDPC, ranging from 90-650, in season two, 
lower than the susceptible check CACUKE, whose AUDPC was 700 in season two. Only 6 genotypes from the ALBW set namely ALBW-4, ALBW-100, ALBW-106, ALBW-173, ALBW-174 and ALBW-204 showed R or MR infection types in both seasons (Table 2).

Among the 47 genotypes in the EPCBW set, the AUDPC ranged from 20-230 in season one and 0-605 in season two. EPCBW genotypes depicted lower rAUDPC, than ALBW genotypes ranging between 0-46 in season one and 0-86 in season two. The EPCBW genotypes had the highest number of intermediate infection type (M) in (twenty two genotypes) (Table 2). Among the PCHP accessions, AUDPC ranged from 78-303 in season one and 0-415 in season two lower than those in the ALBW set. The rFRS ranged from 14-35 in season one and 0-42 in season two. Fourteen of the 19 PCHPBW genotypes evaluated (74\%) showed moderately susceptible to susceptible infection types.

Across all test material thirty five wheat genotypes depicted the pseudo black chaff (PBC) on the spikes and the necks of the plants in season two.

\subsection{Correlation Coefficients among Disease Parameters and Agronomic Traits}

The Pearson Correlation coefficients considered between pairs of the respective disease parameters were highly significant $(p \leq 0.05)$ (Table 3). Among the agronomic traits, negative values for Pearson correlation coefficient were observed with respect to stem rust severity. Specifically, Pearson's correlation coefficients with rust severity were $-0.0475,-0.0253$ and -0.3401 respectively for plant height, spikelet length and TKW (Table 4). This indicated that as the stem rust severities increased, this had negative effects on the agronomic traits. There were significant differences in the relationships between spikelet length and plant height; thousand kernel weight and spikelet length and stem rust and thousand kernel weights $(p \leq 0.05)$. The p-values of all the other agronomic traits were not significantly different from each other (Table 4$)$.

Table 1. ANOVA table highlighting levels of significance observed among various disease and agronomic parameters

\begin{tabular}{lllllllll}
\hline SOURCE & Df & AUDPC & rAUDPC & ACI & rFRS & TKW $(\mathrm{g})$ & PH $(\mathrm{cm})$ & SL $(\mathrm{cm})$ \\
\hline Genotype & 316 & $20167^{*}$ & $411.6^{*}$ & $126.7^{*}$ & $320.2^{*}$ & 34.46 & $67.79^{*}$ & $1.441^{*}$ \\
Season & 1 & $37712^{*}$ & $769.6^{*}$ & 83.27 & 360.2 & $4037.23^{*}$ & $687.7^{*}$ & 0.3222 \\
Genotype by Season & 316 & $13136^{*}$ & $268.1^{*}$ & $69.09^{*}$ & $208.4^{*}$ & 33.08 & 18.02 & 0.5211 \\
Residual & 316 & 8943 & 182.5 & 52.38 & 134.6 & 27.72 & 33.22 & 0.8384 \\
Total & 633 & & & & & & &
\end{tabular}

Note. ${ }^{*}$ Significance at $p \leq 0.05 . \mathrm{df}=$ degree of freedom; AUDPC $=$ area under the disease progress curve; rAUDPC = relative area under the disease progress curve; $\mathrm{ACI}=$ average coefficient of infection; $\mathrm{rFRS}=$ relative final rust severity; TKW = thousand kernel weight in grams; $\mathrm{PH}=$ plant height; $\mathrm{SL}=$ spikelet length in centimeters.

Table 2. Means among different disease and agronomic traits parameters among 316 tested genotypes

\begin{tabular}{|c|c|c|c|c|c|c|c|c|c|c|c|c|}
\hline \multirow{2}{*}{ Genotype } & \multirow{2}{*}{$\mathrm{ACI}$} & \multirow{2}{*}{ AUDPC } & \multirow{2}{*}{ rAUDPC } & \multirow{2}{*}{ rFRS } & \multicolumn{2}{|c|}{ Stem rust season 1} & \multicolumn{2}{|c|}{ Stem rust season 2} & \multirow{2}{*}{ TKW (g) } & \multirow{2}{*}{$\mathrm{PH}(\mathrm{cm})$} & \multirow{2}{*}{$\mathrm{SL}(\mathrm{cm})$} & \multirow{2}{*}{ PBC } \\
\hline & & & & & Severity & FR & Severity & FR & & & & \\
\hline AL BW-1 & 6 & 114 & 32.6 & 11.8 & 10 & MSS & 10 & MR & 32.2 & 77.4 & 8.5 & - \\
\hline AL BW-2 & 10 & 134 & 38.3 & 14.7 & 5 & MSS & 20 & MSS & 37.6 & 73.7 & 9.2 & - \\
\hline AL BW-3 & 44 & 627.5 & 179.3 & 44.1 & 60 & MSS & 15 & MSS & 37.3 & 73.5 & 8.5 & - \\
\hline AL BW-4 & 4 & 47.5 & 13.6 & 5.9 & 10 & MSS & 0 & $\mathrm{TR}$ & 39.3 & 61.5 & 7.2 & - \\
\hline AL BW-5 & 9 & 105 & 30 & 14.7 & 20 & MSS & 5 & MR & 36.8 & 71.5 & 6.5 & - \\
\hline AL BW-6 & 8 & 141.5 & 40.4 & 11.8 & 10 & MSS & 10 & MS & 44.9 & 72.3 & 6.8 & - \\
\hline AL BW-7 & 11 & 170 & 48.6 & 17.6 & 20 & MSS & 10 & M & 33.8 & 76.9 & 7.7 & - \\
\hline AL BW-8 & 18 & 256.5 & 73.3 & 26.5 & 15 & MSS & 30 & MSS & 35.1 & 81.7 & 8.9 & - \\
\hline AL BW-9 & 18 & 229 & 65.4 & 26.5 & 15 & MSS & 30 & MSS & 39.2 & 76.5 & 7.6 & - \\
\hline AL BW-10 & 20 & 285 & 81.4 & 29.4 & 30 & MSS & 20 & MSS & 35.4 & 73.5 & 9.2 & - \\
\hline AL BW-11 & 18 & 247.5 & 70.7 & 26.5 & 5 & MSS & 40 & MSS & 29.8 & 80.9 & 8.8 & - \\
\hline AL BW-12 & 19.5 & 274 & 78.3 & 26.5 & 15 & $\mathrm{~S}$ & 30 & MSS & 31.6 & 86.5 & 8.6 & - \\
\hline
\end{tabular}




\begin{tabular}{|c|c|c|c|c|c|c|c|c|c|c|c|c|}
\hline \multirow{2}{*}{ Genotype } & \multirow{2}{*}{$\mathrm{ACI}$} & \multirow{2}{*}{ AUDPC } & \multirow{2}{*}{ rAUDPC } & \multirow{2}{*}{ rFRS } & \multicolumn{2}{|c|}{ Stem rust season 1} & \multicolumn{2}{|c|}{ Stem rust season 2} & \multirow{2}{*}{ TKW (g) } & \multirow{2}{*}{$\mathrm{PH}(\mathrm{cm})$} & \multirow{2}{*}{$\mathrm{SL}(\mathrm{cm})$} & \multirow{2}{*}{$\mathrm{PBC}$} \\
\hline & & & & & Severity & FR & Severity & FR & & & & \\
\hline AL BW-13 & 24 & 360 & 102.9 & 35.3 & 30 & MSS & 30 & MSS & 33.1 & 72.4 & 8.6 & - \\
\hline AL BW-14 & 28 & 397.5 & 113.6 & 41.2 & 20 & MSS & 50 & MSS & 35 & 64.5 & 7.8 & - \\
\hline AL BW-15 & 30 & 419 & 119.7 & 44.1 & 25 & MSS & 50 & MSS & 33 & 79.5 & 7.6 & - \\
\hline AL BW-16 & 40 & 521.5 & 149 & 52.9 & 40 & $\mathrm{~S}$ & 50 & MSS & 24.4 & 75.2 & 8.1 & - \\
\hline AL BW-17 & 20 & 275 & 78.6 & 29.4 & 10 & MSS & 40 & MSS & 35.5 & 78.4 & 8.4 & - \\
\hline AL BW-18 & 22 & 286.5 & 81.9 & 32.4 & 15 & MSS & 40 & MSS & 28.2 & 81 & 7.3 & - \\
\hline AL BW-19 & 20 & 275.5 & 78.7 & 29.4 & 20 & MSS & 30 & MSS & 27.3 & 83.9 & 8.5 & - \\
\hline AL BW-20 & 20 & 294 & 84 & 29.4 & 10 & MSS & 40 & MSS & 29.6 & 73.3 & 8.7 & - \\
\hline AL BW-21 & 18 & 320 & 91.4 & 26.5 & 5 & MSS & 40 & MSS & 33.5 & 89.5 & 8.4 & - \\
\hline AL BW-22 & 15 & 191.5 & 54.7 & 23.5 & 10 & M & 30 & MSS & 29.6 & 68.3 & 8.8 & - \\
\hline AL BW-23 & 9.5 & 151.5 & 43.3 & 14.7 & 5 & M & 20 & MS & 29.7 & 74.4 & 8.1 & - \\
\hline AL BW-24 & 16 & 191.5 & 54.7 & 23.5 & 10 & MSS & 30 & MSS & 33.6 & 71.5 & 8.1 & - \\
\hline AL BW-25 & 14 & 190 & 54.3 & 20.6 & 5 & MSS & 30 & MSS & 29.7 & 79 & 8.5 & - \\
\hline AL BW-26 & 13.5 & 192 & 54.9 & 20.6 & 5 & M & 30 & MSS & 32.2 & 78.4 & 9.3 & - \\
\hline AL BW-27 & 28 & 406.5 & 116.1 & 41.2 & 20 & MSS & 50 & MSS & 33 & 80.2 & 7.9 & - \\
\hline AL BW-28 & 16 & 272.5 & 77.9 & 23.5 & 20 & MSS & 20 & MSS & 28.3 & 76.7 & 7.9 & - \\
\hline AL BW-29 & 10 & 170.5 & 48.7 & 14.7 & 5 & MSS & 20 & MSS & 31.3 & 69.7 & 9.5 & - \\
\hline AL BW-30 & 19 & 294 & 84 & 29.4 & 10 & M & 40 & MSS & 36.9 & 74 & 9 & - \\
\hline AL BW-31 & 16 & 200 & 57.1 & 23.5 & 10 & MSS & 30 & MSS & 29.4 & 73.5 & 8.1 & - \\
\hline AL BW-32 & 16 & 227.5 & 65 & 23.5 & 20 & MSS & 20 & MSS & 34.5 & 81.2 & 8.8 & - \\
\hline AL BW-33 & 12 & 216.5 & 61.9 & 17.6 & 20 & MSS & 10 & MSS & 28.3 & 77.9 & 9.2 & - \\
\hline AL BW-34 & 15 & 219 & 62.6 & 23.5 & 30 & MSS & 10 & M & 32.6 & 84.3 & 9.3 & - \\
\hline AL BW-35 & 16 & 245 & 70 & 23.5 & 25 & MSS & 15 & MS & 35.5 & 83.8 & 7.7 & - \\
\hline AL BW-36 & 28 & 305.5 & 87.3 & 35.3 & 40 & $\mathrm{~S}$ & 20 & MS & 37 & 69.7 & 9 & - \\
\hline AL BW-37 & 11 & 153 & 43.7 & 17.6 & 20 & MSS & 10 & M & 30.1 & 67.5 & 8.7 & - \\
\hline AL BW-38 & 14 & 207.5 & 59.3 & 20.6 & 20 & MSS & 15 & MS & 34.2 & 66 & 10.2 & - \\
\hline AL BW-39 & 8 & 124 & 35.4 & 14.7 & 15 & MSS & 10 & MR & 36.7 & 75.7 & 8.5 & + \\
\hline AL BW-40 & 22 & 331.5 & 94.7 & 32.4 & 15 & MSS & 40 & MSS & 28.1 & 74.5 & 8.1 & - \\
\hline AL BW-41 & 18 & 229 & 65.4 & 26.5 & 15 & MSS & 30 & MSS & 33.6 & 77.5 & 8.8 & - \\
\hline AL BW-42 & 16 & 227.5 & 65 & 23.5 & 10 & MSS & 30 & MSS & 32.8 & 81.7 & 9.8 & - \\
\hline AL BW-43 & 12.5 & 226.5 & 64.7 & 20.6 & 20 & MSS & 15 & M & 31.8 & 71.3 & 8.6 & - \\
\hline AL BW-44 & 18 & 229 & 65.4 & 26.5 & 15 & MSS & 30 & MSS & 32.3 & 76.2 & 8.9 & - \\
\hline AL BW-45 & 4.3 & 55 & 15.7 & 6.5 & 10 & M & 10 & MS & 38 & 80.7 & 8.8 & - \\
\hline AL BW-46 & 6 & 104 & 29.7 & 8.8 & 5 & MS & 10 & MS & 29.8 & 81.5 & 7.9 & - \\
\hline AL BW-47 & 22 & 312.5 & 89.3 & 32.4 & 15 & MSS & 40 & MSS & 29.6 & 80.3 & 8.6 & - \\
\hline AL BW-48 & 5.5 & 104 & 29.7 & 8.8 & 5 & M & 10 & MS & 32.2 & 77 & 9.6 & - \\
\hline AL BW-49 & 22 & 276.5 & 79 & 29.4 & 40 & $\mathrm{~S}$ & 10 & MR & 35.7 & 71.4 & 9.3 & + \\
\hline AL BW-50 & 14 & 255.5 & 73 & 23.5 & 20 & M & 20 & MSS & 34.2 & 81.2 & 8.7 & - \\
\hline AL BW-51 & 6 & 131.5 & 37.6 & 8.8 & 10 & MSS & 5 & MS & 37.2 & 76.9 & 8.7 & - \\
\hline AL BW-52 & 9 & 114 & 32.6 & 11.8 & 10 & S & 10 & MSS & 34.4 & 72.5 & 9.2 & - \\
\hline AL BW-53 & 5 & 104 & 29.7 & 8.8 & 5 & MSS & 10 & $\mathrm{M}$ & 35.3 & 80 & 8.1 & - \\
\hline AL BW-54 & 12 & 161.5 & 46.1 & 17.6 & 10 & MSS & 20 & MSS & 33.4 & 76 & 8.6 & - \\
\hline AL BW-55 & 5.5 & 112.5 & 32.1 & 8.8 & 5 & M & 10 & MS & 37.7 & 84.5 & 8.2 & - \\
\hline AL BW-56 & 38 & 490 & 140 & 55.9 & 25 & MSS & 70 & MSS & 25.8 & 73.5 & 8.5 & - \\
\hline AL BW-57 & 3.5 & 131.5 & 37.6 & 8.8 & 10 & MR & 5 & M & 37.7 & 78.2 & 9.9 & + \\
\hline AL BW-58 & 14 & 171.5 & 49 & 20.6 & 15 & MSS & 20 & MSS & 26.9 & 73.2 & 8.5 & - \\
\hline AL BW-59 & 8 & 95 & 27.1 & 11.8 & 10 & MS & 10 & MS & 39.6 & 79.5 & 7.8 & - \\
\hline AL BW-60 & 13 & 134 & 38.3 & 17.6 & 10 & $\mathrm{~S}$ & 20 & MSS & 35.6 & 80.7 & 9.1 & - \\
\hline AL BW-61 & 4.5 & 114 & 32.6 & 11.8 & 5 & M & 15 & MR & 34.2 & 85.5 & 8.8 & + \\
\hline AL BW-62 & 8 & 122.5 & 35 & 11.8 & 10 & MSS & 10 & MSS & 28.4 & 77.4 & 8.6 & - \\
\hline
\end{tabular}




\begin{tabular}{|c|c|c|c|c|c|c|c|c|c|c|c|c|}
\hline \multirow{2}{*}{ Genotype } & \multirow{2}{*}{$\mathrm{ACI}$} & \multirow{2}{*}{ AUDPC } & \multirow{2}{*}{ rAUDPC } & \multirow{2}{*}{ rFRS } & \multicolumn{2}{|c|}{ Stem rust season 1} & \multicolumn{2}{|c|}{ Stem rust season 2} & \multirow{2}{*}{ TKW (g) } & \multirow{2}{*}{$\mathrm{PH}(\mathrm{cm})$} & \multirow{2}{*}{$\mathrm{SL}(\mathrm{cm})$} & \multirow{2}{*}{$\mathrm{PBC}$} \\
\hline & & & & & Severity & FR & Severity & FR & & & & \\
\hline AL BW-63 & 12 & 180 & 51.4 & 17.6 & 10 & MSS & 20 & MSS & 36.9 & 79.3 & 8 & - \\
\hline AL BW-64 & 13.5 & 190 & 54.3 & 20.6 & 5 & M & 30 & MSS & 30.2 & 80.4 & 8.4 & - \\
\hline AL BW-65 & 22 & 295.5 & 84.4 & 32.4 & 15 & MSS & 40 & MSS & 33 & 66.9 & 8.7 & - \\
\hline AL BW-66 & 7 & 141.5 & 40.4 & 11.8 & 10 & MSS & 10 & M & 29.5 & 75.7 & 9.8 & - \\
\hline AL BW-67 & 8 & 114 & 32.6 & 11.8 & 15 & MSS & 5 & MS & 31 & 71.9 & 9 & - \\
\hline AL BW-68 & 24 & 314 & 89.7 & 35.3 & 30 & MSS & 30 & MSS & 32.1 & 77 & 8.8 & - \\
\hline AL BW-69 & 18 & 275 & 78.6 & 26.5 & 15 & MSS & 30 & MSS & 36.3 & 81.4 & 7.3 & - \\
\hline AL BW-70 & 17 & 218 & 62.3 & 20.6 & 30 & $\mathrm{~S}$ & 5 & MSS & 34 & 77.5 & 8.8 & - \\
\hline AL BW-71 & 7 & 141.5 & 40.4 & 11.8 & 10 & M & 10 & MSS & 29.8 & 73.4 & 7.1 & - \\
\hline AL BW-72 & 4.5 & 112.5 & 32.1 & 8.8 & 10 & M & 5 & M & 35.7 & 62.5 & 8.1 & - \\
\hline AL BW-73 & 5 & 86.5 & 24.7 & 11.8 & 10 & M & 10 & MR & 37.5 & 84.5 & 8.3 & + \\
\hline AL BW-74 & 8 & 105.5 & 30.1 & 11.8 & 15 & MSS & 5 & MS & 37.2 & 71.9 & 8.1 & - \\
\hline AL BW-75 & 20 & 294 & 84 & 29.4 & 35 & MSS & 15 & MSS & 26.8 & 76.5 & 8.3 & - \\
\hline AL BW-76 & 12 & 161.5 & 46.1 & 17.6 & 20 & MSS & 10 & MSS & 26.7 & 86.5 & 7.8 & - \\
\hline AL BW-77 & 13 & 209 & 59.7 & 20.6 & 25 & MSS & 10 & M & 28.9 & 74.2 & 9.2 & - \\
\hline AL BW-78 & 14 & 190 & 54.3 & 20.6 & 15 & MSS & 20 & MSS & 25.4 & 79.9 & 10.2 & - \\
\hline AL BW-79 & 8.5 & 163 & 46.6 & 20.6 & 15 & M & 20 & MR & 33.3 & 80.9 & 7.9 & + \\
\hline AL BW-80 & 12.5 & 190.5 & 54.4 & 20.6 & 20 & MSS & 15 & M & 32 & 71.9 & 10.2 & - \\
\hline AL BW-81 & 16 & 209 & 59.7 & 23.5 & 20 & MSS & 20 & MSS & 26.6 & 66.7 & 9 & - \\
\hline AL BW-82 & 9 & 151.5 & 43.3 & 14.7 & 15 & MSS & 10 & M & 38.2 & 68.8 & 8.6 & - \\
\hline AL BW-83 & 14 & 217.5 & 62.1 & 20.6 & 15 & MSS & 20 & MSS & 32.1 & 77 & 8.7 & - \\
\hline AL BW-84 & 14 & 218 & 62.3 & 20.6 & 15 & MSS & 20 & MSS & 28.4 & 76.3 & 8.1 & - \\
\hline AL BW-85 & 23 & 266.5 & 76.1 & 29.4 & 40 & $\mathrm{~S}$ & 10 & M & 35 & 81.7 & 8.5 & - \\
\hline AL BW-86 & 28 & 341.5 & 97.6 & 35.3 & 40 & $\mathrm{~S}$ & 20 & MSS & 39.7 & 85.4 & 7.7 & - \\
\hline AL BW-87 & 18 & 303 & 86.6 & 29.4 & 20 & M & 30 & MSS & 28.4 & 82.5 & 8.9 & - \\
\hline AL BW-88 & 10 & 153 & 43.7 & 17.6 & 20 & M & 10 & MS & 26 & 83.3 & 9.7 & - \\
\hline AL BW-89 & 12 & 255 & 72.9 & 23.5 & 20 & M & 20 & M & 31.4 & 78.9 & 8 & - \\
\hline AL BW-90 & 12 & 216.5 & 61.9 & 17.6 & 20 & MSS & 10 & MSS & 35.6 & 73.4 & 8.3 & - \\
\hline AL BW-91 & 10 & 179 & 51.1 & 14.7 & 20 & MS & 5 & MS & 30 & 87.2 & 9.3 & - \\
\hline AL BW-92 & 5 & 76.5 & 21.9 & 8.8 & 10 & M & 5 & MS & 31.7 & 71.3 & 9.6 & - \\
\hline AL BW-93 & 3.5 & 58 & 16.6 & 5.9 & 5 & M & 5 & M & 29.3 & 77.9 & 9.1 & - \\
\hline AL BW-94 & 5 & 78 & 22.3 & 11.8 & 10 & M & 10 & MR & 31.8 & 81.3 & 8.2 & + \\
\hline AL BW-95 & 3.5 & 95.5 & 27.3 & 8.8 & 5 & M & 10 & MR & 34.1 & 73 & 8.7 & + \\
\hline AL BW-96 & 5.5 & 54.5 & 15.6 & 8.8 & 5 & M & 10 & MS & 36.9 & 73 & 8.1 & - \\
\hline AL BW-97 & 3.5 & 68 & 19.4 & 8.8 & 10 & MR & 5 & M & 34.2 & 71.7 & 8.5 & + \\
\hline AL BW-98 & 3.5 & 104 & 29.7 & 8.8 & 10 & MR & 5 & M & 47.4 & 72 & 8.1 & + \\
\hline AL BW-99 & 2.5 & 47.5 & 13.6 & 5.9 & 5 & MR & 5 & M & 30.7 & 64.7 & 8.4 & + \\
\hline AL BW-100 & 3 & 40 & 11.4 & 5.9 & 10 & M & 0 & $\mathrm{TR}$ & 30.2 & 72.3 & 9.1 & - \\
\hline AL BW-101 & 8 & 105.5 & 30.1 & 11.8 & 10 & MSS & 10 & MS & 32.6 & 79.8 & 8.9 & - \\
\hline AL BW-102 & 12 & 227.5 & 65 & 23.5 & 20 & M & 20 & M & 32.6 & 86.5 & 10.1 & - \\
\hline AL BW-103 & 10 & 124 & 35.4 & 14.7 & 5 & MSS & 20 & MSS & 28.7 & 71.3 & 8.7 & - \\
\hline AL BW-104 & 12 & 171.5 & 49 & 17.6 & 15 & MSS & 15 & MS & 29.1 & 81.7 & 9.8 & - \\
\hline AL BW-105 & 8 & 141.5 & 40.4 & 11.8 & 10 & MSS & 10 & MS & 28.6 & 76.3 & 8 & - \\
\hline AL BW-106 & 8 & 123 & 35.1 & 11.8 & 20 & MSS & 0 & $\mathrm{TR}$ & 34.5 & 62.8 & 7.5 & - \\
\hline AL BW-107 & 5.5 & 85 & 24.3 & 8.8 & 10 & MSS & 5 & M & 36.6 & 71.2 & 8.5 & - \\
\hline AL BW-108 & 11 & 197.5 & 56.4 & 17.6 & 10 & M & 20 & MSS & 30.9 & 61.5 & 9.1 & - \\
\hline AL BW-109 & 20 & 320 & 91.4 & 29.4 & 20 & MSS & 30 & MSS & 32.2 & 82 & 8.6 & - \\
\hline AL BW-110 & 14 & 218 & 62.3 & 20.6 & 30 & MSS & 5 & MS & 37.8 & 83.8 & 8.9 & - \\
\hline AL BW-111 & 7 & 141.5 & 40.4 & 11.8 & 10 & M & 10 & MS & 33.4 & 81.5 & 9.2 & - \\
\hline AL BW-112 & 12 & 189 & 54 & 17.6 & 25 & MSS & 5 & MS & 30 & 84.7 & 8.3 & - \\
\hline
\end{tabular}




\begin{tabular}{|c|c|c|c|c|c|c|c|c|c|c|c|c|}
\hline \multirow{2}{*}{ Genotype } & \multirow{2}{*}{$\mathrm{ACI}$} & \multirow{2}{*}{ AUDPC } & \multirow{2}{*}{ rAUDPC } & \multirow{2}{*}{ rFRS } & \multicolumn{2}{|c|}{ Stem rust season 1} & \multicolumn{2}{|c|}{ Stem rust season 2} & \multirow{2}{*}{ TKW (g) } & \multirow{2}{*}{$\mathrm{PH}(\mathrm{cm})$} & \multirow{2}{*}{$\mathrm{SL}(\mathrm{cm})$} & \multirow{2}{*}{$\mathrm{PBC}$} \\
\hline & & & & & Severity & FR & Severity & FR & & & & \\
\hline AL BW-113 & 13.5 & 181.5 & 51.9 & 20.6 & 30 & MSS & 5 & $\mathrm{M}$ & 36.3 & 79.8 & 9.2 & - \\
\hline AL BW-114 & 20 & 230.5 & 65.9 & 29.4 & 30 & MSS & 20 & MSS & 32.6 & 71.4 & 9.7 & - \\
\hline AL BW-115 & 23 & 257.5 & 73.6 & 29.4 & 30 & S & 20 & MSS & 30.3 & 76.7 & 10 & - \\
\hline AL BW-116 & 8.5 & 134 & 38.3 & 14.7 & 15 & M & 10 & MS & 33.4 & 71.5 & 9.8 & - \\
\hline AL BW-117 & 12.5 & 209 & 59.7 & 20.6 & 15 & M & 20 & MSS & 31.9 & 80 & 8.2 & - \\
\hline AL BW-118 & 16 & 229 & 65.4 & 23.5 & 20 & MSS & 20 & MSS & 26.7 & 76 & 10.6 & - \\
\hline AL BW-119 & 14 & 190.5 & 54.4 & 20.6 & 20 & MSS & 15 & MSS & 29.2 & 72.3 & 8.4 & - \\
\hline AL BW-120 & 10 & 170.5 & 48.7 & 14.7 & 20 & MSS & 5 & MS & 33.6 & 79.9 & 9.8 & - \\
\hline AL BW-121 & 16 & 200.5 & 57.3 & 23.5 & 20 & MSS & 20 & MSS & 26.3 & 72.7 & 9.9 & - \\
\hline AL BW-122 & 8 & 151.5 & 43.3 & 14.7 & 10 & MR & 15 & MSS & 30.2 & 84.5 & 8.6 & - \\
\hline AL BW-123 & 16 & 219 & 62.6 & 23.5 & 10 & MSS & 30 & MSS & 32.9 & 75 & 9.1 & - \\
\hline AL BW-124 & 4 & 104 & 29.7 & 8.8 & 5 & MS & 10 & MR & 27.8 & 74.2 & 9 & + \\
\hline AL BW-125 & 10 & 132.5 & 37.9 & 14.7 & 15 & MSS & 10 & MSS & 36.3 & 76.2 & 8.4 & - \\
\hline AL BW-126 & 14 & 226.5 & 64.7 & 20.6 & 25 & MSS & 10 & MSS & 31.9 & 83.7 & 9.5 & - \\
\hline AL BW-127 & 16 & 228 & 65.1 & 23.5 & 25 & MSS & 15 & MSS & 28.3 & 82.7 & 9.2 & - \\
\hline AL BW-128 & 11 & 180.5 & 51.6 & 17.6 & 20 & MSS & 10 & M & 36.2 & 81.2 & 7.9 & - \\
\hline AL BW-129 & 20 & 247.5 & 70.7 & 29.4 & 20 & MSS & 30 & MSS & 37.8 & 88.3 & 8.1 & - \\
\hline AL BW-130 & 6.5 & 160.5 & 45.9 & 11.8 & 15 & M & 5 & MS & 31.1 & 85.5 & 9.1 & - \\
\hline AL BW-131 & 7 & 141.5 & 40.4 & 11.8 & 10 & M & 10 & MSS & 34.1 & 79.8 & 9.2 & - \\
\hline AL BW-132 & 12 & 216.5 & 61.9 & 17.6 & 20 & MSS & 10 & MSS & 32.5 & 76.9 & 8.6 & - \\
\hline AL BW-133 & 9 & 199 & 56.9 & 17.6 & 20 & M & 10 & M & 36.5 & 77.7 & 8.9 & + \\
\hline AL BW-134 & 11 & 207.5 & 59.3 & 17.6 & 10 & M & 20 & MSS & 28.7 & 79.5 & 7.7 & - \\
\hline AL BW-135 & 9 & 245.5 & 70.1 & 17.6 & 20 & M & 10 & M & 34.1 & 94 & 9.8 & - \\
\hline AL BW-136 & 9.5 & 218 & 62.3 & 20.6 & 25 & M & 10 & MR & 29.8 & 88.5 & 10.5 & + \\
\hline AL BW-137 & 24.5 & 304 & 86.9 & 32.4 & 25 & $\mathrm{~S}$ & 30 & MSS & 30.8 & 77.2 & 9.1 & - \\
\hline AL BW-138 & 14.5 & 180 & 51.4 & 17.6 & 25 & S & 5 & MS & 29.5 & 82.2 & 8.7 & - \\
\hline AL BW-139 & 10 & 143 & 40.9 & 14.7 & 15 & MSS & 10 & MS & 32.9 & 78.5 & 8.2 & - \\
\hline AL BW-140 & 16 & 200.5 & 57.3 & 23.5 & 30 & MSS & 10 & MS & 29.4 & 73.7 & 9.3 & - \\
\hline AL BW-141 & 14 & 173 & 49.4 & 20.6 & 25 & MSS & 10 & MS & 30.4 & 67.2 & 8 & - \\
\hline AL BW-142 & 5.5 & 76.5 & 21.9 & 8.8 & 5 & M & 10 & MS & 37.5 & 72.5 & 9.6 & - \\
\hline AL BW-143 & 7 & 114 & 32.6 & 11.8 & 10 & M & 10 & MSS & 33.8 & 71.7 & 8.4 & - \\
\hline AL BW-144 & 32 & 435 & 124.3 & 41.2 & 40 & $\mathrm{~S}$ & 30 & MSS & 31.2 & 83.5 & 9.5 & - \\
\hline AL BW-145 & 9.5 & 153 & 43.7 & 14.7 & 5 & M & 20 & MSS & 30.4 & 84.7 & 10.4 & - \\
\hline AL BW-146 & 10 & 151.5 & 43.3 & 14.7 & 15 & MSS & 10 & MS & 34.1 & 85 & 8.7 & - \\
\hline AL BW-147 & 24.5 & 321.5 & 91.9 & 32.4 & 25 & $\mathrm{~S}$ & 30 & MSS & 35.6 & 72.9 & 7.5 & - \\
\hline AL BW-148 & 26 & 360.5 & 103 & 35.3 & 20 & $\mathrm{~S}$ & 40 & MSS & 32.3 & 76.9 & 9.8 & - \\
\hline AL BW-149 & 12 & 180.5 & 51.6 & 17.6 & 10 & MS & 20 & MSS & 35 & 75.7 & 8.5 & - \\
\hline AL BW-150 & 16 & 237.5 & 67.9 & 23.5 & 20 & MSS & 20 & MSS & 31.1 & 81.5 & 10 & - \\
\hline AL BW-151 & 6 & 133 & 38 & 11.8 & 10 & M & 10 & M & 30 & 73.7 & 9.7 & - \\
\hline AL BW-152 & 3.5 & 85.5 & 24.4 & 5.9 & 5 & MSS & 5 & M & 36 & 77.9 & 9.6 & - \\
\hline AL BW-153 & 4 & 75 & 21.4 & 5.9 & 5 & MSS & 5 & MS & 38.4 & 83.5 & 10.3 & - \\
\hline AL BW-154 & 6 & 105.5 & 30.1 & 11.8 & 10 & M & 10 & M & 39.4 & 83.7 & 9.7 & - \\
\hline AL BW-155 & 4 & 95.5 & 27.3 & 8.8 & 10 & M & 5 & MR & 34.8 & 79.4 & 8.2 & + \\
\hline AL BW-156 & 7 & 124 & 35.4 & 11.8 & 15 & MSS & 5 & MR & 30 & 70.5 & 8.6 & - \\
\hline AL BW-157 & 15.5 & 218 & 62.3 & 20.6 & 25 & $\mathrm{~S}$ & 10 & M & 30.4 & 76.7 & 8.4 & - \\
\hline AL BW-158 & 6 & 133 & 38 & 11.8 & 15 & M & 5 & M & 29.1 & 76.4 & 9.4 & + \\
\hline AL BW-159 & 10 & 114 & 32.6 & 14.7 & 15 & MSS & 10 & MS & 35.5 & 76.2 & 10.6 & - \\
\hline AL BW-160 & 5 & 112.5 & 32.1 & 8.8 & 10 & M & 5 & MSS & 36.6 & 82 & 8.3 & - \\
\hline AL BW-161 & 8.5 & 143 & 40.9 & 14.7 & 15 & M & 10 & MSS & 36.4 & 80.4 & 7.9 & - \\
\hline AL BW-162 & 7.5 & 151.5 & 43.3 & 14.7 & 15 & M & 10 & M & 40.1 & 89.5 & 9.5 & + \\
\hline
\end{tabular}




\begin{tabular}{|c|c|c|c|c|c|c|c|c|c|c|c|c|}
\hline \multirow{2}{*}{ Genotype } & \multirow{2}{*}{$\mathrm{ACI}$} & \multirow{2}{*}{ AUDPC } & \multirow{2}{*}{ rAUDPC } & \multirow{2}{*}{ rFRS } & \multicolumn{2}{|c|}{ Stem rust season 1} & \multicolumn{2}{|c|}{ Stem rust season 2} & \multirow{2}{*}{ TKW (g) } & \multirow{2}{*}{$\mathrm{PH}(\mathrm{cm})$} & \multirow{2}{*}{$\mathrm{SL}(\mathrm{cm})$} & \multirow{2}{*}{$\mathrm{PBC}$} \\
\hline & & & & & Severity & FR & Severity & FR & & & & \\
\hline AL BW-163 & 5.5 & 85 & 24.3 & 8.8 & 5 & $\mathrm{M}$ & 10 & MSS & 37.2 & 63.4 & 7.4 & - \\
\hline AL BW-164 & 6.5 & 105.5 & 30.1 & 11.8 & 15 & M & 5 & MS & 38.4 & 88.2 & 8.5 & - \\
\hline AL BW-165 & 13.5 & 200.5 & 57.3 & 20.6 & 30 & MSS & 5 & M & 32.7 & 80.2 & 8.4 & - \\
\hline AL BW-166 & 11.5 & 197.5 & 56.4 & 17.6 & 25 & MSS & 5 & M & 33.3 & 79.8 & 8.5 & - \\
\hline AL BW-167 & 11.5 & 199 & 56.9 & 20.6 & 25 & M & 10 & MS & 27.5 & 74.2 & 8.3 & - \\
\hline AL BW-168 & 31 & 371.5 & 106.1 & 41.2 & 30 & S & 40 & MSS & 30.7 & 81.2 & 9 & - \\
\hline AL BW-169 & 18 & 116 & 33.1 & 23.5 & 20 & S & 20 & MSS & 36.4 & 87.5 & 9 & - \\
\hline AL BW-170 & 7 & 133 & 38 & 11.8 & 10 & M & 10 & MS & 30.7 & 91.7 & 9.7 & - \\
\hline AL BW-171 & 5.5 & 76.5 & 21.9 & 8.8 & 10 & MSS & 5 & M & 37.7 & 88.9 & 9.9 & - \\
\hline AL BW-172 & 5 & 104 & 29.7 & 8.8 & 10 & M & 5 & MSS & 37.2 & 84.2 & 8.9 & - \\
\hline AL BW-173 & 1.5 & 29 & 8.3 & 2.9 & 5 & M & 0 & $\mathrm{TR}$ & 38.9 & 74.7 & 8.4 & - \\
\hline AL BW-174 & 3 & 66.5 & 19 & 5.9 & 10 & M & 0 & $\mathrm{TR}$ & 35.1 & 71.8 & 8.7 & - \\
\hline AL BW-175 & 6 & 76.5 & 21.9 & 8.8 & 10 & MSS & 5 & MSS & 39.2 & 64.4 & 9.2 & - \\
\hline AL BW-176 & 15.5 & 163 & 46.6 & 20.6 & 15 & $\mathrm{~S}$ & 20 & MSS & 35.7 & 72.9 & 10.2 & - \\
\hline AL BW-177 & 3 & 85.5 & 24.4 & 5.9 & 5 & M & 5 & M & 36.4 & 74 & 9.2 & - \\
\hline AL BW-178 & 18 & 275 & 78.6 & 26.5 & 15 & MSS & 30 & MSS & 30.5 & 81.7 & 9.7 & - \\
\hline AL BW-179 & 8 & 114 & 32.6 & 11.8 & 10 & MSS & 10 & MS & 31.9 & 85.3 & 8.8 & - \\
\hline AL BW-180 & 7 & 114 & 32.6 & 11.8 & 10 & MS & 10 & M & 34.4 & 81 & 8.7 & - \\
\hline AL BW-181 & 9 & 190.5 & 54.4 & 17.6 & 10 & M & 20 & M & 33.7 & 73.5 & 8.2 & + \\
\hline AL BW-182 & 6.5 & 187.5 & 53.6 & 11.8 & 15 & M & 5 & MS & 30.9 & 69.9 & 9 & - \\
\hline AL BW-183 & 14 & 217.5 & 62.1 & 20.6 & 15 & MSS & 20 & MSS & 26.7 & 79.5 & 9.4 & - \\
\hline AL BW-184 & 9 & 161.5 & 46.1 & 17.6 & 10 & M & 20 & M & 32.2 & 86.7 & 8.5 & - \\
\hline AL BW-185 & 12 & 180.5 & 51.6 & 17.6 & 20 & MSS & 10 & MSS & 30.5 & 85.2 & 9.8 & - \\
\hline AL BW-186 & 7.5 & 105.5 & 30.1 & 11.8 & 15 & MSS & 5 & M & 32.7 & 74.8 & 9.6 & - \\
\hline AL BW-187 & 16 & 246.5 & 70.4 & 23.5 & 20 & MSS & 20 & MSS & 41.4 & 86.3 & 9.3 & - \\
\hline AL BW-188 & 16 & 219 & 62.6 & 23.5 & 20 & MSS & 20 & MSS & 32.5 & 77.5 & 8.9 & - \\
\hline AL BW-189 & 24 & 331.5 & 94.7 & 35.3 & 30 & MSS & 30 & MSS & 31.3 & 71.5 & 9 & - \\
\hline AL BW-190 & 16 & 227.5 & 65 & 23.5 & 20 & MSS & 20 & MSS & 26.2 & 79.7 & 10.8 & - \\
\hline AL BW-191 & 35 & 455 & 130 & 47.1 & 30 & $\mathrm{~S}$ & 50 & MSS & 25 & 79 & 10.2 & - \\
\hline AL BW-192 & 37.5 & 511.5 & 146.1 & 50 & 35 & $\mathrm{~S}$ & 50 & MSS & 26.9 & 72.9 & 8.4 & - \\
\hline AL BW-193 & 7 & 133 & 38 & 11.8 & 10 & MS & 10 & M & 34.4 & 79 & 8.4 & - \\
\hline AL BW-194 & 5 & 95.5 & 27.3 & 8.8 & 5 & MS & 10 & M & 34.6 & 75.2 & 8.6 & - \\
\hline AL BW-195 & 8 & 114 & 32.6 & 11.8 & 10 & MSS & 10 & MSS & 34.8 & 78 & 8.4 & - \\
\hline AL BW-196 & 24 & 367.5 & 105 & 35.3 & 10 & MS & 50 & MSS & 27.1 & 81.2 & 9.2 & - \\
\hline AL BW-197 & 5.5 & 104 & 29.7 & 8.8 & 5 & M & 10 & MSS & 31.9 & 78.9 & 9.3 & - \\
\hline AL BW-198 & 11 & 134 & 38.3 & 17.6 & 10 & M & 20 & MSS & 28.8 & 72.5 & 8.5 & - \\
\hline AL BW-199 & 11 & 125.5 & 35.9 & 17.6 & 10 & M & 20 & MSS & 29.1 & 87.8 & 8 & - \\
\hline AL BW-200 & 14 & 164 & 46.9 & 23.5 & 20 & M & 20 & MSS & 32.2 & 71.7 & 7.4 & - \\
\hline AL BW-201 & 16.5 & 229 & 65.4 & 26.5 & 15 & M & 30 & MSS & 36.2 & 77.4 & 7.7 & - \\
\hline AL BW-202 & 12.5 & 209 & 59.7 & 20.6 & 15 & $15 \mathrm{M}$ & 20 & MSS & 34.4 & 77.5 & 9.9 & - \\
\hline AL BW-203 & 5 & 114 & 32.6 & 11.8 & 10 & M & 10 & MR & 30.9 & 80 & 10.2 & + \\
\hline AL BW-204 & 4.5 & 76.5 & 21.9 & 8.8 & 15 & M & 0 & $\mathrm{TR}$ & 40 & 76.7 & 8.4 & - \\
\hline AL BW-205 & 11 & 153 & 43.7 & 17.6 & 10 & M & 20 & MSS & 32.6 & 75 & 8.4 & - \\
\hline AL BW-206 & 17 & 265 & 75.7 & 26.5 & 5 & MR & 40 & MSS & 36.8 & 73.8 & 7.3 & - \\
\hline AL BW-207 & 14 & 245 & 70 & 23.5 & 20 & M & 20 & MSS & 32.7 & 67.5 & 8.3 & - \\
\hline AL BW-208 & 8 & 122.5 & 35 & 11.8 & 10 & MSS & 10 & MSS & 33.8 & 68.2 & 9 & - \\
\hline AL BW-209 & 9 & 151.5 & 43.3 & 14.7 & 15 & MSS & 10 & M & 33 & 74 & 8.3 & - \\
\hline AL BW-210 & 7 & 114 & 32.6 & 11.8 & 15 & MSS & 5 & MR & 35.7 & 65 & 8.4 & - \\
\hline AL BW-211 & 10 & 160 & 45.7 & 14.7 & 15 & MSS & 10 & MSS & 31.7 & 78.2 & 10.2 & - \\
\hline AL BW-212 & 16 & 161.5 & 46.1 & 23.5 & 20 & MSS & 20 & MSS & 32.9 & 72.4 & 9.7 & - \\
\hline
\end{tabular}




\begin{tabular}{|c|c|c|c|c|c|c|c|c|c|c|c|c|}
\hline \multirow{2}{*}{ Genotype } & \multirow{2}{*}{$\mathrm{ACI}$} & \multirow{2}{*}{ AUDPC } & \multirow{2}{*}{ rAUDPC } & \multirow{2}{*}{ rFRS } & \multicolumn{2}{|c|}{ Stem rust season 1} & \multicolumn{2}{|c|}{ Stem rust season 2} & \multirow{2}{*}{ TKW (g) } & \multirow{2}{*}{$\mathrm{PH}(\mathrm{cm})$} & \multirow{2}{*}{$\mathrm{SL}(\mathrm{cm})$} & \multirow{2}{*}{$\mathrm{PBC}$} \\
\hline & & & & & Severity & FR & Severity & FR & & & & \\
\hline AL BW-213 & 20 & 285 & 81.4 & 29.4 & 20 & MSS & 30 & MSS & 33.7 & 76.3 & 9.3 & - \\
\hline AL BW-214 & 28 & 389 & 111.1 & 41.2 & 30 & MSS & 40 & MSS & 35.8 & 77.2 & 8.8 & - \\
\hline AL BW-215 & 32 & 329 & 94 & 47.1 & 30 & MSS & 50 & MSS & 27.3 & 75.9 & 8.6 & - \\
\hline AL BW-216 & 26 & 452.5 & 129.3 & 41.2 & 20 & M & 50 & MSS & 29.5 & 77.2 & 8.1 & - \\
\hline AL BW-217 & 12 & 209 & 59.7 & 23.5 & 20 & M & 20 & M & 32.6 & 73.9 & 8.1 & + \\
\hline AL BW-218 & 4 & 131.5 & 37.6 & 8.8 & 10 & M & 5 & MR & 37.5 & 76.5 & 7.2 & + \\
\hline AL BW-219 & 6.5 & 143 & 40.9 & 14.7 & 15 & M & 10 & MSS & 40.2 & 80.9 & 9.7 & + \\
\hline AL BW-220 & 6 & 114 & 32.6 & 11.8 & 10 & M & 10 & M & 37.7 & 83.5 & 9.4 & - \\
\hline AL BW-221 & 4.5 & 104 & 29.7 & 8.8 & 5 & M & 10 & M & 36.2 & 74.2 & 9 & - \\
\hline AL BW-222 & 3.5 & 76.5 & 21.9 & 8.8 & 5 & M & 10 & MR & 35.9 & 74.2 & 8.2 & + \\
\hline AL BW-223 & 6.5 & 143 & 40.9 & 14.7 & 15 & M & 10 & MR & 29.8 & 78 & 8.6 & + \\
\hline AL BW-224 & 12 & 180.5 & 51.6 & 17.6 & 15 & MS & 15 & MSS & 36.5 & 70 & 8.7 & - \\
\hline AL BW-225 & 14 & 209 & 59.7 & 20.6 & 15 & MSS & 20 & MSS & 30.6 & 67.7 & 8.2 & - \\
\hline AL BW-226 & 18 & 246.5 & 70.4 & 26.5 & 15 & MSS & 30 & MSS & 26.6 & 76 & 8.8 & - \\
\hline AL BW-227 & 16 & 190 & 54.3 & 23.5 & 20 & MS & 20 & MSS & 26.5 & 78.5 & 10.3 & - \\
\hline AL BW-228 & 15 & 220.5 & 63 & 23.5 & 10 & M & 30 & MSS & 34.5 & 74.4 & 10 & - \\
\hline AL BW-229 & 20 & 275.5 & 78.7 & 29.4 & 20 & MSS & 30 & MSS & 24.9 & 80 & 8.9 & - \\
\hline AL BW-230 & 7.5 & 95 & 27.1 & 11.8 & 5 & M & 15 & MSS & 40.9 & 79.7 & 8.1 & - \\
\hline AL BW-231 & 21 & 246.5 & 70.4 & 26.5 & 30 & $\mathrm{~S}$ & 15 & MSS & 35.3 & 80.2 & 8.1 & - \\
\hline AL BW-232 & 14 & 209 & 59.7 & 20.6 & 5 & MSS & 30 & MSS & 28.3 & 84 & 8.7 & - \\
\hline AL BW-233 & 16 & 191.5 & 54.7 & 23.5 & 10 & MSS & 30 & MSS & 34.1 & 78.2 & 10 & - \\
\hline AL BW-234 & 6 & 133 & 38 & 11.8 & 10 & M & 10 & M & 33.6 & 81.7 & 9.2 & - \\
\hline AL BW-235 & 3 & 20 & 5.7 & 5.9 & 5 & M & 5 & M & 33.7 & 80.8 & 8.3 & - \\
\hline AL BW-236 & 2.5 & 66.5 & 19 & 5.9 & 5 & M & 5 & MR & 30.2 & 75.5 & 7.3 & + \\
\hline AL BW-237 & 2.5 & 66.5 & 19 & 5.9 & 5 & M & 5 & MR & 32.6 & 78 & 8.3 & + \\
\hline AL BW-238 & 12 & 170 & 48.6 & 17.6 & 20 & MSS & 10 & MS & 34 & 81.4 & 8 & - \\
\hline AL BW-239 & 16 & 246.5 & 70.4 & 23.5 & 20 & MSS & 20 & MSS & 38.7 & 76.2 & 10.4 & - \\
\hline AL BW-240 & 15 & 237.5 & 67.9 & 23.5 & 10 & M & 30 & MSS & 32.2 & 74 & 9.8 & - \\
\hline AL BW-241 & 6 & 133 & 38 & 11.8 & 10 & M & 10 & M & 38 & 73.7 & 8.1 & - \\
\hline AL BW-242 & 5.5 & 85 & 24.3 & 8.8 & 10 & MSS & 5 & M & 40.8 & 74.7 & 8.9 & - \\
\hline AL BW-243 & 5 & 85 & 24.3 & 8.8 & 10 & M & 5 & MS & 32.1 & 76.2 & 8.6 & - \\
\hline AL BW-244 & 10 & 151.5 & 43.3 & 14.7 & 15 & MSS & 10 & MSS & 29.1 & 77.8 & 9.4 & - \\
\hline AL BW-245 & 16 & 246.5 & 70.4 & 23.5 & 20 & MSS & 20 & MSS & 30.4 & 68 & 9.7 & - \\
\hline AL BW-246 & 4.5 & 104 & 29.7 & 8.8 & 5 & M & 10 & M & 30.9 & 78.9 & 9.2 & - \\
\hline AL BW-247 & 8 & 114 & 32.6 & 11.8 & 10 & MSS & 10 & MS & 33.7 & 71.2 & 9.7 & - \\
\hline AL BW-248 & 6 & 85 & 24.3 & 8.8 & 10 & MSS & 5 & MSS & 31.2 & 79.4 & 12.1 & - \\
\hline AL BW-249 & 2.5 & 66.5 & 19 & 5.9 & 5 & M & 5 & MR & 30.4 & 77.7 & 8.4 & + \\
\hline AL BW-250 & 5 & 95.5 & 27.3 & 8.8 & 10 & M & 5 & MS & 33.2 & 72.5 & 8.8 & - \\
\hline EPC BW-251 & 4 & 68 & 19.4 & 8.8 & 10 & M & 5 & MR & 33.5 & 70.3 & 8.4 & + \\
\hline EPC BW-252 & 11 & 180 & 51.4 & 17.6 & 10 & M & 20 & MSS & 25.9 & 77.9 & 10.1 & - \\
\hline EPC BW-253 & 15 & 274 & 78.3 & 23.5 & 10 & M & 30 & MSS & 29.6 & 77.9 & 9.8 & - \\
\hline EPC BW-254 & 13.5 & 162.5 & 46.4 & 20.6 & 5 & M & 30 & MSS & 26.6 & 79.3 & 9.7 & - \\
\hline EPC BW-255 & 15 & 191.5 & 54.7 & 23.5 & 10 & M & 30 & MSS & 26.7 & 78.2 & 9.7 & - \\
\hline EPC BW-256 & 12 & 285 & 81.4 & 29.4 & 10 & M & 40 & M & 30 & 76.4 & 10.3 & + \\
\hline EPC BW-257 & 8 & 141.5 & 40.4 & 11.8 & 10 & MSS & 10 & MS & 25.8 & 70.8 & 8.7 & - \\
\hline EPC BW-258 & 4.5 & 104 & 29.7 & 8.8 & 10 & M & 5 & M & 25.4 & 77.9 & 9.2 & + \\
\hline EPC BW-259 & 6 & 141.5 & 40.4 & 11.8 & 10 & M & 10 & M & 26 & 79.7 & 10.6 & + \\
\hline EPC BW-260 & 10 & 151.5 & 43.3 & 14.7 & 20 & MSS & 5 & MS & 33 & 72.9 & 8.4 & - \\
\hline EPC BW-261 & 8 & 114 & 32.6 & 11.8 & 20 & MSS & 0 & $\mathrm{TR}$ & 30.1 & 83.5 & 9.5 & - \\
\hline EPC BW-262 & 4 & 76.5 & 21.9 & 8.8 & 10 & M & 5 & MR & 37.4 & 69.5 & 10.5 & + \\
\hline
\end{tabular}




\begin{tabular}{|c|c|c|c|c|c|c|c|c|c|c|c|c|}
\hline \multirow{2}{*}{ Genotype } & \multirow{2}{*}{ ACI } & \multirow{2}{*}{ AUDPC } & \multirow{2}{*}{ rAUDPC } & \multirow{2}{*}{ rFRS } & \multicolumn{2}{|c|}{ Stem rust season 1} & \multicolumn{2}{|c|}{ Stem rust season 2} & \multirow{2}{*}{ TKW (g) } & \multirow{2}{*}{$\mathrm{PH}(\mathrm{cm})$} & \multirow{2}{*}{$\mathrm{SL}(\mathrm{cm})$} & \multirow{2}{*}{$\mathrm{PBC}$} \\
\hline & & & & & Severity & FR & Severity & FR & & & & \\
\hline EPC BW-263 & 4 & 76.5 & 21.9 & 8.8 & 10 & $\mathrm{M}$ & 5 & MR & 33.4 & 75.5 & 7.9 & + \\
\hline EPC BW-264 & 18 & 284 & 81.1 & 26.5 & 15 & MSS & 30 & MSS & 25.9 & 80.3 & 8.4 & - \\
\hline EPC BW-265 & 18 & 246.5 & 70.4 & 26.5 & 25 & MSS & 20 & MSS & 29.5 & 83.8 & 10.7 & - \\
\hline EPC BW-266 & 5.5 & 85 & 24.3 & 8.8 & 5 & M & 10 & MSS & 33.8 & 71.7 & 9.2 & - \\
\hline EPC BW-267 & 28 & 425 & 121.4 & 41.2 & 20 & MSS & 50 & MSS & 25 & 73.4 & 8.3 & - \\
\hline EPC BW-268 & 22 & 312.5 & 89.3 & 32.4 & 15 & MSS & 40 & MSS & 28.3 & 74.8 & 9.7 & - \\
\hline EPC BW-269 & 18 & 246.5 & 70.4 & 26.5 & 15 & MSS & 30 & MSS & 32.9 & 79.2 & 9.2 & - \\
\hline EPC BW-270 & 12 & 254 & 72.6 & 17.6 & 20 & MSS & 10 & MSS & 38.1 & 74.4 & 7.7 & - \\
\hline EPC BW-271 & 12 & 216.5 & 61.9 & 17.6 & 20 & $\mathrm{~S}$ & 10 & MR & 32.3 & 75 & 8.8 & - \\
\hline EPC BW-272 & 12 & 189 & 54 & 17.6 & 20 & MSS & 10 & MSS & 32.2 & 78.7 & 8.3 & - \\
\hline EPC BW-273 & 6 & 122.5 & 35 & 11.8 & 10 & MSS & 10 & MR & 31.5 & 82 & 8.5 & - \\
\hline EPC BW-274 & 9.5 & 151.5 & 43.3 & 14.7 & 20 & MSS & 5 & M & 28.5 & 76.7 & 9.8 & - \\
\hline EPC BW-275 & 5.5 & 141.5 & 40.4 & 11.8 & 15 & MSS & 5 & MR & 35 & 69.7 & 8.7 & + \\
\hline EPC BW-276 & 9 & 170 & 48.6 & 14.7 & 20 & MSS & 5 & MR & 37.2 & 84.9 & 7.9 & - \\
\hline EPC BW-277 & 10 & 160 & 45.7 & 14.7 & 20 & MSS & 5 & MS & 34.6 & 77.9 & 8 & - \\
\hline EPC BW-278 & 5 & 104 & 29.7 & 8.8 & 10 & M & 5 & MS & 33 & 69.3 & 9.3 & - \\
\hline EPC BW-279 & 4 & 47.5 & 13.6 & 5.9 & 5 & MSS & 5 & MSS & 25.9 & 80.7 & 9.9 & - \\
\hline EPC BW-280 & 4 & 66.5 & 19 & 5.9 & 5 & MSS & 5 & MSS & 27 & 73.9 & 10.3 & - \\
\hline EPC BW-281 & 15 & 219 & 62.6 & 23.5 & 10 & M & 30 & MSS & 27.9 & 71.9 & 9.1 & - \\
\hline EPC BW-282 & 26 & 416.5 & 119 & 38.2 & 25 & MSS & 40 & MSS & 25.7 & 73 & 8.5 & - \\
\hline EPC BW-283 & 5 & 85 & 24.3 & 8.8 & 10 & M & 5 & MSS & 32.7 & 82.4 & 9.2 & - \\
\hline EPC BW-284 & 12.5 & 217.5 & 62.1 & 20.6 & 15 & M & 20 & MSS & 28 & 72.3 & 10.2 & - \\
\hline EPC BW-285 & 8 & 133 & 38 & 11.8 & 15 & MSS & 5 & MS & 32.5 & 77.7 & 10 & - \\
\hline EPC BW-286 & 14 & 217.5 & 62.1 & 20.6 & 15 & MSS & 20 & MS & 31.8 & 80.4 & 10.7 & - \\
\hline EPC BW-287 & 8 & 160 & 45.7 & 14.7 & 20 & M & 5 & MS & 41.6 & 79.8 & 8.8 & - \\
\hline EPC BW-288 & 8.5 & 151.5 & 43.3 & 14.7 & 15 & M & 10 & MSS & 32.2 & 82.4 & 9.2 & - \\
\hline EPC BW-289 & 20 & 375 & 107.1 & 29.4 & 20 & MSS & 30 & MSS & 29 & 74.2 & 7.8 & - \\
\hline EPC BW-290 & 15 & 227.5 & 65 & 23.5 & 10 & M & 30 & MSS & 32.1 & 80.2 & 8.2 & - \\
\hline EPC BW-291 & 12.5 & 190 & 54.3 & 20.6 & 15 & M & 20 & MSS & 28.8 & 71.8 & 8.8 & - \\
\hline EPC BW-292 & 12 & 216.5 & 61.9 & 17.6 & 20 & MSS & 10 & MS & 27.8 & 63.8 & 8 & - \\
\hline EPC BW-293 & 10.5 & 161.5 & 46.1 & 17.6 & 15 & M & 15 & MSS & 29.8 & 72.5 & 8.6 & - \\
\hline EPC BW-294 & 9 & 151.5 & 43.3 & 14.7 & 15 & MSS & 10 & M & 28.9 & 77.4 & 9.7 & - \\
\hline EPC BW-295 & 6 & 122.5 & 35 & 11.8 & 20 & M & 0 & $\mathrm{TR}$ & 30.5 & 73.4 & 9.4 & - \\
\hline EPC BW-296 & 8 & 133 & 38 & 11.8 & 15 & MSS & 5 & MS & 31.4 & 80.2 & 8 & - \\
\hline EPC BW-297 & 10 & 124 & 35.4 & 14.7 & 20 & MSS & 5 & MS & 31.3 & 74.7 & 9.9 & - \\
\hline PCHP BW-298 & 12 & 180.5 & 51.6 & 17.6 & 20 & MSS & 10 & MSS & 35.8 & 79.5 & 10.1 & - \\
\hline PCHP BW-299 & 18 & 284 & 81.1 & 26.5 & 25 & MSS & 20 & MSS & 31.3 & 77.5 & 8.2 & - \\
\hline PCHP BW-300 & 18 & 238 & 68 & 26.5 & 15 & MSS & 30 & MSS & 36.4 & 68.4 & 7.9 & - \\
\hline PCHP BW-301 & 14 & 217.5 & 62.1 & 20.6 & 15 & MSS & 20 & MSS & 35.7 & 71.9 & 7.8 & - \\
\hline PCHP BW-302 & 7 & 141.5 & 40.4 & 11.8 & 10 & M & 10 & MSS & 31.8 & 79.4 & 9.2 & - \\
\hline PCHP BW-303 & 16 & 246.5 & 70.4 & 23.5 & 20 & MSS & 20 & MSS & 32.3 & 72.7 & 9.3 & - \\
\hline PCHP BW-304 & 20 & 312.5 & 89.3 & 29.4 & 20 & MSS & 30 & MSS & 30.7 & 83.9 & 11 & - \\
\hline PCHP BW-305 & 14 & 209 & 59.7 & 20.6 & 15 & MSS & 20 & MSS & 34.2 & 74.4 & 10.4 & - \\
\hline PCHP BW-306 & 7 & 141.5 & 40.4 & 11.8 & 10 & M & 10 & MS & 34.6 & 77.4 & 8.9 & - \\
\hline PCHP BW-307 & 8.5 & 151.5 & 43.3 & 14.7 & 15 & M & 10 & MSS & 38.3 & 69.2 & 8.5 & - \\
\hline PCHP BW-308 & 6 & 133 & 38 & 11.8 & 10 & M & 10 & M & 32.7 & 74.2 & 8.7 & + \\
\hline PCHP BW-309 & 4 & 39 & 11.1 & 5.9 & 10 & MSS & 0 & $\mathrm{TR}$ & 29.3 & 74.7 & 9.6 & - \\
\hline PCHP BW-310 & 8 & 114 & 32.6 & 11.8 & 20 & MSS & 0 & $\mathrm{TR}$ & 32.3 & 71 & 8.2 & - \\
\hline PCHP BW-311 & 10 & 151.5 & 43.3 & 14.7 & 20 & MSS & 5 & MS & 36.5 & 77.2 & 7.4 & - \\
\hline PCHP BW-312 & 12 & 197.5 & 56.4 & 17.6 & 20 & MSS & 10 & MSS & 40.6 & 62.5 & 8.6 & - \\
\hline
\end{tabular}




\begin{tabular}{|c|c|c|c|c|c|c|c|c|c|c|c|c|}
\hline \multirow{2}{*}{ Genotype } & \multirow{2}{*}{$\mathrm{ACI}$} & \multirow{2}{*}{ AUDPC } & \multirow{2}{*}{ rAUDPC } & \multirow{2}{*}{ rFRS } & \multicolumn{2}{|c|}{ Stem rust season 1} & \multicolumn{2}{|c|}{ Stem rust season 2} & \multirow{2}{*}{ TKW (g) } & \multirow{2}{*}{$\mathrm{PH}(\mathrm{cm})$} & \multirow{2}{*}{$\mathrm{SL}(\mathrm{cm})$} & \multirow{2}{*}{$\mathrm{PBC}$} \\
\hline & & & & & Severity & FR & Severity & FR & & & & \\
\hline PCHP BW-313 & 10 & 151.5 & 43.3 & 14.7 & 15 & MSS & 10 & MS & 40.9 & 81 & 9.4 & - \\
\hline PCHP BW-314 & 12 & 197.5 & 56.4 & 17.6 & 20 & MSS & 10 & MS & 34.9 & 78.5 & 10.1 & - \\
\hline PCHP BW-315 & 9 & 179 & 51.1 & 17.6 & 20 & M & 10 & M & 34 & 78 & 8.7 & + \\
\hline PCHP BW-316 & 18 & 238 & 68 & 26.5 & 25 & MSS & 20 & MSS & 36.4 & 67.5 & 9.1 & - \\
\hline CACUKE & 56 & 350 & 100 & 100 & 90 & MSS & 100 & MSS & 20.2 & 60.7 & 9.5 & - \\
\hline Grand Mean & 12.3 & 187 & 26.7 & 22.9 & 15.7 & & 20 & & 32.5 & 76.8 & 8.8 & \\
\hline LSD & 10.7 & 139.8 & 19.97 & 17.15 & 12 & & 16 & & 7.7 & 8.2 & 1.4 & \\
\hline CV (\%) & 8.8 & 5.6 & 5.6 & 5.7 & 5.7 & & 7.2 & & 16.2 & 7.5 & 10.4 & \\
\hline
\end{tabular}

Note. ACI $=$ average coefficient of infection; AUDPC $=$ area under the disease progress curve; rAUDPC $=$ relative area under the disease progress curve; $\mathrm{rFRS}=$ relative final rust severity; Disease Severity based on Modified Cobb's (0-100\%) scale (Peterson et al., 1948); IT = Infection Type based on (Roelfs et al., 1992); TR = trace, $\mathrm{R}=$ resistant, $\mathrm{MR}=$ moderately resistant, $\mathrm{RMR}=$ resistant to moderately resistant, MRMS $(\mathrm{M})=$ moderately resistant to moderately susceptible, MSS = moderately susceptible to susceptible, $\mathrm{MS}=$ moderately susceptible and $\mathrm{S}=$ susceptible; TKW $=$ thousand kernel weight in grams; $\mathrm{PH}=$ Plant Height in centimeters; SL $=$ Spike length in centimeters TKW $=$ thousand kernel weight; $\mathrm{PBC}=$ Pseudo Black Chaff, $(+)$ predictive of Sr2 gene, (-) absence of Sr 2 gene; LSD = Least Significant Difference CV (\%) = Percentage Coefficient of Variation.

Table 3. Pearson's correlation coefficient for the disease parameters among the wheat genotypes across seasons

\begin{tabular}{lllll}
\hline & ACI & AUDPC & rAUDPC & rFRS \\
\hline ACI & - & & & \\
AUDPC & $0.9242^{* * *}$ & - & & \\
rAUDPC & $0.9242^{* * *}$ & $1.0000^{* * *}$ & - & \\
rFRS & $0.9745^{* * *}$ & $0.8982^{* * *}$ & $0.8982^{* * *}$ & - \\
\hline
\end{tabular}

Note. ${ }^{* * *}$ : large positive relationship between the variables at $p \leq 0.05 ; \mathrm{ACI}=$ average coefficient of infection; AUDPC $=$ area under the disease progress curve; rAUDPC $=$ relative area under the disease progress curve; $\mathrm{rFRS}=$ relative final rust severity.

Table 4. Pearson's correlation coefficient for the different agronomic traits among the wheat genotypes across seasons

\begin{tabular}{llllll}
\hline & Number of tillers & Plant height & Spikelet length & Thousand kernel weight & Stem rust \\
\hline Number of tillers & - & & & & \\
Plant height & 0.0475 & - & & & \\
Spikelet length & -0.0924 & 0.1472 & - & & \\
Thousand kernel weight & 0.0848 & 0.0269 & -0.2167 & - & - \\
Stem rust severity & 0.0559 & -0.0475 & -0.0253 & -0.3401 & \\
\hline
\end{tabular}

\section{Discussion}

Seasonal effects of temperature and precipitation on disease development were discernible. In season one when the temperatures were higher and the precipitation was lower, the infection rate was apparently lower. Abiotic stresses such embodied in high temperatures and drought in general induce the plant defense pathway leading to increased plant resistance to stem rust (Mittler, 2006). During the second season in which relatively lower temperatures and high precipitation were recorded, higher disease pressure was noted. Besides supporting vigorous plant growth, providing increased surface area for spore landing and infection, lower temperatures and "free water" through precipitation favors rust infection process per se. Similar findings have been extensively reported in rust epidemiology literature e.g. as highlighted in GRDC (2011). The present results underscores that KALRO-Njoro sits in an environment conducive to stem rust proliferation. In the larger East African region stem rust epidemics are driven by a combination of factors including favorable weather conditions for the disease, volunteer host plants, and presence of a green crop of wheat at any one time, also referred to as a "green bridge" 
that enhance survival build-up and spread of the stem rust pathogen through the seasons. In combination with growing of stem rust susceptible varieties especially among the small scale resource poor farmers, these factors have aggravated the Ug99 threat in the region leading to seasonal stem rust outbreaks (GRDC, 2011).

Different reactions to stem rust observed between the genotypes suggested that the material had diverse genetic backgrounds. It can be inferred that the six genotypes namely ALBW-106, ALBW-204, EPCBW-261, EPCBW-295, PCHPBW-309 and PCHPBW-310 that only showed trace response to the diseases (TR) with no visible stem rust infections could either be carrying single effective major genes or a combination of those. Singh et al. (2005) reported that a combination of 4-5 minor effect genes with race non-specific responses provided near immunity reaction to leaf rust. Accordingly, the 6 highly resistant lines could also be harboring a combination of minor effect genes. Leonard and Szabo (2005) suggested that the presence of effective major genes in a variety limit infection process by triggering necrosis of the host cells in the neighborhood of the infective structures.

Among the 250 ALBW accessions, 22 and 26 genotypes were susceptible during season one and two respectively, while 135 genotypes showed moderately susceptible (MS) response. Their severities however were low, compared to the susceptible check variety CACUKE whose final severity was $90 \mathrm{~S}$ and $100 \mathrm{~S}$ during season one and two respectively. The ALBW are among old varieties bred between the 70s and the 80s by CIMMYT Mexico (Kohli \& Rajaram, 1988). The high frequency of MS to S genotypes among the ALBW genotypes suggested presence of ineffective stem rust resistance genes in their backgrounds, probably $\operatorname{SrTmp}, \operatorname{Sr} 24, \operatorname{Sr} 31$, to which the current family of Ug99 races are highly virulent. Results on previous rust resistance studies on genotypes within the ALBW set are consistent with the present study. For instance, Chaves et al. (2011) reported high frequency of moderately susceptible to susceptible infection types within Brazilian genotypes with aluminium tolerance backgrounds. Notably, 19 genotypes among the ALBW set showed resistant to moderately resistant (RMR) infection types. Among those, 6 genotypes (ALBW-4, ALBW-100, ALBW-106, ALBW-173, ALBW-174 and ALBW-204) showed resistant (R) infection type with no visible or compatible interaction between the host genotypes and the stem rust fungus despite the heavy disease pressure in season two. This suggests that these lines could be carrying stem rust resistance genes which are still effective against the Ug99 and its variants.

Among the 47 EPCBW genotypes, nearly half showed intermediate infection type (M), and relatively low severities ranging from $5 \%$ to $20 \%$ during both seasons implying that these genotypes could be containing effective stem rust resistance genes in their backgrounds. Despite, some level of disease, which in fact suggests incomplete resistance, these lines produced plump grain with no apparent stem rust associated yield loss.

While over $70 \%$ of the 19 PCHPBW genotypes evaluated during both seasons, showed MSS infection types, those nonetheless depicted lower severities. Moreover, those genotypes expressed the pseudo black chaff phenotype, implying the presence of the adult plant resistance gene-Sr2. These lines could serve as useful genetic resources in breeding for durable resistance to the prevailing stem rust races, especially when combined with effective major genes. Such a strategy will not only counteract the wheat yield losses currently common in many wheat growing zones of Kenya but equally important, it will counter the rapid evolution of new stem rust races due to delayed step-wise mutations triggered by over cultivation of single resistance varieties over extensive acreages and across seasons (Tsilo et al., 2010).

Across the two seasons of evaluation, 14 genotypes exhibiting dwarfing traits also had relatively high TKW values. The TKW is considered a "yield component" and a good proxy for yield potential of a genotype (Dill-Macky et al., 1990). The semi-dwarf stature of these genotypes suggested presence of dwarfing genes (Rht), which reduce height. Semi dwarf genotypes unlike the traditionally taller varieties are tolerant to lodging, and hence are more responsive to high nitrogen-fertilization and irrigated cropping systems especially under intensive management. Development of semi-dwarf types of wheats was initiated at CIMMYT by Norman Borlaug in the early 1960s through crosses made between the double-dwarf Japanese cultivar Norin and taller breeding lines (Gale et al., 1981; Kihara, 1984). A report by Sayre et al. (1997) indicated that there has been an annual increase in yield by $1 \%$ among semi-dwarf wheat varieties due to incorporation of dwarfing genes in their backgrounds.

The large negative correlation between TKW and stem rust can be attributed to the fact that the fungus damages the vascular system of the susceptible host plant extensively limiting transportation of water and nutrients from the soil to the developing kernel and other organs as well as interfering with translocation of photosynthates, which leads to shrivelled grains (Singh et al., 2006; Everts et al., 2001). Similar results have been reported by numerous previous research groups (Tadesse et al., 2010; Taye et al., 2015). Among the highly susceptible varieties, the endosperm barely forms and resultant grains are invariably completely shrivelled. 


\section{Conclusion}

The six exotic wheat genotypes with high resistance to stem rust could be used as donors for introgression of resistance to the adapted Kenyan wheat backgrounds. This will also help improve Kenyan germplasm with regard to aluminum tolerance and micronutrient fortification (Velu \& Singh, 2013). The 35 genotypes with low MSS response and which also showed presence of PBC could be integrated in the Durable Rust Resistance Wheat (DRRW) pipeline to develop durable sources of resistance to stem rust. Further greenhouse studies involving seedlings coupled with marker assisted selection needs be carried out to identify the exact genes conferring the resistance to stem rust among the exotic varieties. Inheritance studies could also be done among the elite wheat genotypes to elucidate the exact genes and their effects especially in conditioning the stem rust resistance. This will ensure the effective utilization of the resistance sources in the wheat breeding program through their deployment into adapted but susceptible wheat varieties.

\section{Acknowledgements}

This publication was made possible through support provided by Alliance for a Green Revolution in Africa (AGRA). The opinions expressed herein are those of the author(s) and do not necessarily reflect the views of AGRA. My heartfelt gratitude also goes to the Borlaug Global Rust Initiative (BGRI) under the Durable Rust Resistant Wheat (DRRW) project, KALRO-Njoro, my supervisors and other technicians for assisting me in setting up my experiments.

\section{References}

Admassu, B., Lind, V., Friedt, W., \& Ordon, F. (2009). Virulence Analysis of Puccinia graminis f. sp. Tritici Populations in Ethiopia with Special Consideration of Ug99. Plant Pathology, 58(2), 362-369. http://dx.doi.org/10.1111/j.1365-3059.2008.01976.x

Beard, C., Jayasena, K., Thomas, G., \& Loughman, R. (2006). Managing stem rust of wheat. Plant Pathology, Department of Agriculture, Western Australia. Farmnote, 73. Retrieved from https://www.agric.wa.gov.au

Chaves, M. S., Martinelli, J., da Silva, P. R., Scagliusi, S., \& Brammer, S. (2011). Surveillance of Race Ug99 in Brazil and the Search for Effective Resistance (pp. 2-23). Paper presented at the Embrapa Trigo CPACT.

Dill-Macky, R., Rees, R. G., \& Platz, G. J. (1990). Stem Rust Epidemics and Their Effects on Grain Yield and Quality in Australian Barley Cultivars. Crop and Pasture Science, 41(6), 1057-1063. http://dx.doi.org/ 10.1071/AR9901057

Everts, K. L., Leath, S., \& Finney, P. L. (2001). Impact of Powdery Mildew and Leaf Rust on Milling and Baking Quality of Soft Red Winter Wheat. Plant Disease, 85(4), 4. http://dx.doi.org/10.1094/ PDIS.2001.85.4.423

FAO. (2016). Agricultural commodities profiles and relevant WTO negotiations isssues. Economic and Social Development Department. Retrievd September 1, 2016, from http://www.fao.org/economic/ess/ess-home/en

Gale, M. D., Marshall, G. A., \& Rao, M. V. (1981). A Classification of the Norin 10 and Tom Thumb Dwarfing Genes in British, Mexican, Indian and Other Hexaploid Bread Wheat Varieties. Euphytica, 30(2), 355-361. http://dx.doi.org/10.1007/BF00033997

Grain Research and Development Cooperation (GRDC). (2011). Stem rust of wheat fact sheet. Seasonal conditions drive outbreaks. Retrieved from http:// www.grdc.com.au/rustlinks

Hodson, D. P. (2015). Field Survey Protocols and Tools. Paper presented at the 2015 SAARC Wheat Rust Training Course. CIMMYT-Ethiopia.

Jin, Y., Szabo, L. J., Pretorius, Z. A., Singh, R. P., Ward, R., \& Fetch, J. T. (2008). Detection of virulence to resistance gene Sr24 within race TTKS of Puccinia graminis f. sp. tritici. Plant Disease, 92(6), 923-926. http://dx.doi.org/10.1094/PDIS-92-6-0923

Kihara, H. (1984). Origin and History of 'Daruma', A Parental Variety of Norin 10. In S. Sakamoto (Ed.), Proceedings of the 6th International Wheat Genetics Symposium. Plant Germplasm Institute, University of Kyoto, Kyoto, Japan.

Kohli, M. M., \& Rajaram, S. (1988). Review of Brasilian/CIMMYT collaboration 1974-1986. In D. G. Tanner, M. van Ginkel, \& W. Mwangi (Eds.), Wheat Breeding for Acid Soils. Sixth Regional Wheat Workshop for Eastern, Central and Southern Africa. Mexico. D.F.: CIMMYT.

Leonard, K. J., \& Szabo, L. J. (2005). Stem Rust of Small Grains and Grasses Caused by Puccinia graminis. Molecular Plant Pathology, 6(2), 99-111. http://dx.doi.org/10.1111/J.1364-3703.2004.00273.x 
McIntosh, R. A. (1988). The role of specific genes in breeding for durable stem rust resistance in wheat and triticale. In N. W. Simmonds \& R. Sanjaya (Eds.). Breeding strategies for resistance to the rusts of wheat (pp. 1-9). CIMMYT, Mexico.

McIntosh, R. A., Dubcovsky, J., Rogers, W. J., Morris, C., Appels, R., Xia, X. C., \& Azul, B. (2014). Catalogue of Gene Symbols for Wheat: 2013-2014.

Mittler, R. (2006). Abiotic Stress, the Field Environment and Stress Combination. Trends in Plant Science, 11(1), 15-19. http://dx.doi.org/10.1016/j.tplants.2005.11.002

Nazari, K., Mafi, M., Yahyaoui, A., Singh, R. P., \& Park, R. F. (2009). Detection of Wheat Stem Rust (Puccinia graminis f. sp. tritici) Race TTKSK (Ug99) in Iran. Plant Disease, 93(3), 317-317. http://dx.doi.org/ 10.1094/PDIS-93-3-0317B

Njau, P. N., Jin, Y., Huerta-Espino, J., Keller, B., \& Singh, R. P. (2010). Identification and Evaluation of Sources of Resistance to Stem Rust race Ug99 in Wheat. Plant Disease, 94(4), 413-419. http://dx.doi.org/10.1094/ PDIS-94-4-0413

Njau, P. N., Wanyera, R., Macharia, G. K., Singh, J. M. R., \& Keller, B. (2009). Resistance in Kenyan bread wheat to recent eastern African isolate of stem rust, Puccinia graminis f. sp. tritici, Ug99. Journal of Plant Breeding and Crop Science, 1(2), 022-027. Retrieved from http://www.academicjournals.org/jpbcs

Ooro, P. A., Bor, P. K., Amadi, D. O. K., Tenywa, J. S., Joubert, G. D., Marais, D., ... Nampala, M. P. (2009). Evaluation of wheat genotypes for improved drought tolerance through increased seedling vigour. 9th African Crop Science, Conference Proceedings, Cape Town, South Africa, 28 September-2 October 2009. Retrieved from https://www.cabdirect.org/cabdirect/abstract/20133232444

Peterson, R. F., Campbell, A. B., \& Hannah, A. E. (1948). A Diagrammatic Scale for Estimating Rust Intensity on Leaves and Stems of Cereals. Canadian Journal of Research, 26(5), 496-500. http://dx.doi.org/10.1139/ cjr48c-033

Pretorius, Z. A., Singh, R. P., Wagoire, W. W., \& Payne, T. S. (2000). Detection of Virulence to Wheat Stem Rust Resistance Gene Sr31 in Puccinia graminis .f. sp. Tritici in Uganda. Plant Dis., 84, 203. http://dx.doi.org/ 10.1094/PDIS.2000.84.2.203B

Roelfs, A. P., Singh, R. P., \& Saari, E. E. (1992). Rust Diseases of Wheat: Concepts and Methods of Disease Management (2nd ed.). CIMMYT, Mexico, D.F.

Rosegrant, M. W., Agcaoili-Sombilla, M., \& Perez, N. D. (1995). Global Food Projections to 2020: Implications for Investment. International Food Policy Research Institute, Washington, D.C.

Sayre, K. D., Rajaram, S., \& Fischer, R. A. (1997). Yield Potential Progress in Short Bread Wheats in Northwest Mexico. Crop science, 37(1), 36-42. http://dx.doi.org/10.2135/cropsci1997.0011183X003700010006x

Singh, R. P., Hodson, D. P., Jin, Y., Lagudah, E. S., Ayliffe, M. A., Bhavani, S., ... Basnet, B. R. (2015). Emergence and spread of new races of wheat stem rust fungus: Continued threat to food security and prospects of genetic control. Phytopathology, 105(7), 872-884. http://dx.doi.org/10.1094/PHYTO-0115-0030-FI

Singh, R. P., Huerta-Espino, J., \& William, H. M. (2005). Genetics and breeding for durable resistance to leaf and stripe rusts in wheat. Turkish Journal of Agriculture and Forestry, 29(2), 121-127. Retrieved from http://journals.tubitak.gov.tr/agriculture/abstract.htm?id=7487

Singh, R. P., Kinyua, M. G., Wanyera, R., Njau, P., Jin, Y., \& Huerta-Espino, J. (2006). Spread of a Highly Virulent Race of Puccinia graminis Tritici in Eastern Africa: Challenges and Opportunities CAB Reviews. Perspectives in Agriculture, Veterinary Science, Nutrition and Natural Resources, 1, 054. Retrieved from http://dx.doi.org/10.1079/PAVSNNR20061054

Tadesse, K., Ayalew, A., \& Badebo, A. (2010). Effect of Fungicide on the Development of Wheat Stem Rust and Yield of Wheat Varieties in Highlands of Ethiopia. African Crop Science Journal, 18(1). http://dx.doi.org/10.4314/acsj.v18i1.54194

Taye, T., Fininsa, C., \& Woldeab, G. (2015). Yield Variability of Bread Wheat under Wheat Stem Rust Pressure at Bore Field Condition of Southern Oromia. Journal of Agricultural Science and Food Technology, 1(2), 11-15. Retrieved from http://pearlresearchjournals.org/journals/jmbsr/index.html

Tsilo, T. J., Jin, Y., \& Anderson, J. A. (2010). Identification of Flanking Markers for the Stem Rust Resistance Gene Sr6 in Wheat. Crop Science, 50, 1967-1970. http://dx.doi.org/10.2135/cropsci2009.11.0648 
Velu, G., \& Singh, R. P. (2013). Phenotyping for Plant Breeding. In S. K. Pangaluri \& A. A. Kumar (Eds.), Applications of Phenotyping Methods for Crop Improvement. Springer Science \& Business Media.

Wanyera, R., Kinyua, M. G., Jin, Y., \& Singh, R. P. (2006). The Spread of Stem Rust Caused by Puccinia graminis f. sp. tritici, with Virulence on Sr31 in Wheat in Eastern Africa. Plant Disease, 90(1), 113-113. http://dx.doi.org/10.1094/PD-90-0113A

Wilcoxson, R. D., Skovmand, B., \& Atif, A. H. (1975). Evaluation of Wheat Cultivars for Ability to Retard Development of Stem Rust. Annals of Applied Biology, 80(3), 275-281. http://dx.doi.org/10.1111/ j.1744-7348.1975.tb01633.x

\section{Copyrights}

Copyright for this article is retained by the author(s), with first publication rights granted to the journal.

This is an open-access article distributed under the terms and conditions of the Creative Commons Attribution license (http://creativecommons.org/licenses/by/4.0/). 